\title{
Influence of ovariectomy combined with lack of masticatory force in the evolution of periodontal disease
}

\author{
Noala Vicensoto Moreira Milhan', Lilibeth Ferraz de Brito Penna Forte', \\ Luana Marotta Reis de Vasconcelos ${ }^{1}$, Ivan Balducci' ${ }^{2}$, Yasmin Rodarte Carvalho ${ }^{1}$
}

1 Universidade Estadual Paulista - UNESP, School of Dentistry of São José dos Campos, Department of Biosciences and Original Diagnosis,

São José dos Campos, SP, Brasil

2Universidade Estadual Paulista - UNESP, School of Dentistry of São José dos Campos, Department of Restorative Dentistry, São José dos Campos, SP, Brasil

\begin{abstract}
Aim: To evaluate the influence of ovariectomy combined with lack of masticatory force in the evolution of periodontal disease induced in rats. Methods: Forty rats were bilaterally ovariectomized and 40 were submitted to sham ovariectomy. Periodontal disease was induced in the mandibular left first molar and the maxillary left first molar was extracted from half of the rats. The rats were randomly euthanized at $3,7,14$ and 30 days post periodontal disease induction. Serial sections were obtained from the furcation area and stained for histological and histomorphometric analysis. The results of the histomorphometric analysis were statistically analyzed by ANOVA and Tukey tests. Results: The results demonstrated statistically significant differences in the percentage of bone tissue when the variables presence or absence of estrogen $(p=0.020)$ and time of euthanasia $(p=0.002)$ were evaluated. However, the extraction procedure did not significantly affect the percentage of bone tissue $(p=0.598)$. Conclusions: The bone loss resulting from periodontal disease is increased by estrogen deficiency and varies according to the time course of periodontitis. In contrast, masticatory force does not seem to interfere in bone loss derived from periodontal disease.
\end{abstract}

Keywords: ovariectomy; periodontal diseases.

\section{Introduction}

Received for publication: January 15, 2014 Accepted: March 17, 2014

Correspondence to: Noala Vicensoto Moreira Milhan Departamento de Biociência e

Diagnóstico Bucal - UNESP

Avenida Engenheiro Francisco José Longo, 777 Jardim São Dimas - CEP: 12245-000

São José dos Campos, SP, Brasil Phone: +55 1239479384

Fax: +55 1239479010

E-mail: noala.moreira@fosjc.unesp.br
Osteoporosis is a progressive systemic disease that reduces, per unit volume, typically mineralized bone and degrades its microstructure. These factors increase significantly the bone fragility, leading to increased susceptibility to fractures ${ }^{1-3}$. The disease is a public health problem because fractures lead to high costs for the healthcare service due to hospitalizations and associated drugs ${ }^{2,4-5}$.

Osteoporosis and periodontal disease have several common risk factors ${ }^{6}$, including environmental, genetic, dietary and systemic factors ${ }^{7}$. Examples of this are higher prevalence with advancing age ${ }^{8}$, negative impacts of smoking on the development and severity of the disease ${ }^{8-9}$ and deterioration of tissue repair as a result of the disease. In the same way as osteoporosis, periodontal diseases are silent diseases that cause no obvious symptoms until the advanced stages of the disease process, which eventually causes tooth $\operatorname{loss}^{8}$.

Host systemic conditions can determine higher or lower risk of developing periodontitis. These conditions, whether acquired, congenital or hereditary, can modulate the inflammatory response and promote greater individual susceptibility 
to periodontal disease $\mathrm{e}^{9-10}$. One systemic condition that is considered a risk factor for periodontal disease is osteoporosis. The disease usually affects postmenopausal women and may lead to decreased bone mineral density throughout the organism, including promoting alveolar bone loss in the jaw ${ }^{11-15}$.

Several authors have suggested that estrogen deficiency causes changes in alveolar bone that may result in the aggravation of a preexisting periodontal disease ${ }^{1,8,12,14}$. According to Gomes-Filho et al. ${ }^{16}$, postmenopausal women with osteoporosis show a 2.5-fold higher risk of developing periodontal disease than women without osteoporosis. A study conducted by Al Habashneh et al. ${ }^{17}$ showed that osteoporosis is signiûcantly combined with severe alveolar crestal bone loss and prevalence of periodontitis cases in postmenopausal Jordanian women. However, according to Moriya et al. ${ }^{18}$, osteoporosis alone does not promote significant periodontal destruction and thus may not be a major factor in periodontal disease.

According to Patullo et al. ${ }^{19}$, masticatory hypofunction is a major factor in the induction of mandible bone loss and cause harmful effects on bone quality. Elovic et al. ${ }^{20}$ reported that the extraction of maxillary molars promotes a faster deterioration of the structural properties, more rapid decrease in bone mineral density and an important decrease in bone area fraction of the mandible in ovariectomized rats compared with sham ovariectomized rats. Ejiri et al. ${ }^{21}$ observed that occlusal hypofunction can greatly accelerate the fragility of the bone structure in the alveolar bone around extruded teeth. On the other hand, a study by Crespo Vázquez et al. ${ }^{22}$ concluded that the presence of antagonist forces aggravates resorption of the roots in patients with periodontal disease.

Ligature is a great resource to induce periodontal disease in rats, independent of the accompanying diet, as well as to provide material for evaluating the histological characteristics of induced periodontal disease $\mathrm{e}^{23}$. The ligature acts as a subgingival plaque increasing the number of bacteria and induces mechanical trauma in the dentogingival region, leading to diminished tissue integrity, which causes a destructive local inflammatory response in the periodontal region. This inflammatory response leads to apical migration of the junctional epithelium, loosening of periodontal fibers and alveolar bone resorption ${ }^{24-27}$. The anatomy of the dentogingival junction of rat molars is very similar to that in humans, validating experimental studies of inflammatory periodontal disease in this animal model ${ }^{28}$.

The purpose of this study was to evaluate the influence of ovariectomy combined with lack of masticatory force in the evolution of periodontal disease induced in rats.

\section{Material and methods}

\section{Protocol}

Three-month-old female rats (Rattus norvegicus var. Albinus, Wistar) $(\mathrm{n}=80)$, weighing approximately $300 \mathrm{~g}$, provided by the Animal Center of the School of Dentistry of São José dos Campos, Universidade Estadual Paulista (UNESP) were used. This research protocol was approved by the institutional committee for animal experiments (protocol number $01 / 2008-\mathrm{PA} / \mathrm{CEP}$ ). The rats were placed in appropriate cages, and were provided distilled water ad libitum and standard diet. The amount of feed consumed per day was previously determined, based on the mean daily consumption of the rats, to prevent excessive increase in weight ( $22 \mathrm{~g} /$ day).

To simulate an estrogen depression, 40 rats were bilaterally ovariectomized (OVZ) and the other 40 were submitted to sham ovariectomy, to produce surgical stress (SHAM - control group). For this and other procedures the rats were anesthetized via intramuscular injection with $13 \mathrm{mg} / \mathrm{kg}$ of 2-(2,6-xylidine)5,6-dihydro-4H-1,3-thiazine hydrochloride (Rompun; Bayer, Porto Alegre, RS, Brazil), an analgesic, sedative and muscle relaxant, and general anesthetic consisting of $38.5 \mathrm{mg} / \mathrm{kg}$ of ketamine (Dopalen; Agribands do Brasil Ltda, Paulinia, SP, Brazil).

Sixty days after ovariectomy or sham ovariectomy, a ligature was placed around the left mandibular first molar to induce periodontal disease. The ligature was maintained up to euthanasia in each group. On the same day that periodontal disease was induced, the maxillary left first molars were extracted from half the rats of the OVZ and SHAM groups. Thus 4 groups $(n=20)$ were established: SHAM 1, surgical stress and periodontal disease; SHAM 2, surgical stress, periodontal disease and antagonist tooth extraction; OVZ 1, ovariectomy and periodontal disease; and OVZ 2, ovariectomy, periodontal disease and antagonist tooth extraction.

At 3, 7, 14 and 30 days after periodontal disease induction, five rats per group were randomly euthanized for each experimental period. To achieve this, the rats were anesthetized and cardiac perfusion was performed using $4 \%$ formalin. The mandible was removed, followed by removal of any excess of soft tissue, and the bone was fixed in $10 \%$ formaldehyde solution.

\section{Histological and histomorphometric analysis}

Hemisection of the mandible was performed and the left side was immersed in 10\% EDTA solution for demineralization. The specimens were routinely processed for paraffin embedding. Serial sections $(5 \mu \mathrm{m})$ were obtained from the furcation area of the first molar, stained with hematoxylin and eosin (HE) for analysis under light microscopy and then photographed for histomorphometric analysis.

Histological analysis of the furcation area of the left mandibular first molar was performed to verify specific bone tissue features, including osteoblast and osteoclast density, inflammatory infiltration and other signs of periodontal disease.

Histomorphometric measurement was performed to calculate the amount of bone matrix in the furcation area of the left mandibular first molar. This procedure was carried out at x200 magnification using a grid that divided the field into 100 equal parts. The grid was placed over the digitalized image of the histological field. The total number of intersection points between the reticule lines over the bone matrix was counted. The Image J software (NIH, Version Windows 1.4, USA) was used for this procedure. The number of intersections counted 
was divided by the total number of intersections and expressed as a percentage. Two trained and blinded examiners examined each specimen independently.

\section{Statistics analysis}

The results of the histomorphometric analysis were expressed as mean values ( \pm standard deviation). Comparisons among the groups were performed by threeway analysis of variance (ANOVA). The variables considered were presence or absence of estrogen, time of euthanasia and extraction procedure. Post hoc Tukey significant difference analysis was used to determine significant differences among the groups (95\% confidence interval). Values of $p \leq 0.05$ were considered statistically significant.

\section{Results}

The success of ovariectomy was confirmed at necropsy by failure to detect ovarian tissue and by observation of marked atrophy of the uterine horns in the OVZ group.

\section{Histology of the furcation area}

Few differences were observed in the histological features between the OVZ and SHAM groups (presence or absence of estrogen) and the subgroups submitted or not to maxillary left first molar extraction (Figure 1). The most relevant difference was observed between the periods of euthanasia (3, 7, 14 and 30 days).

For the post-periodontal disease induction period of 3 days, immature bone trabeculae with numerous osteoclasts
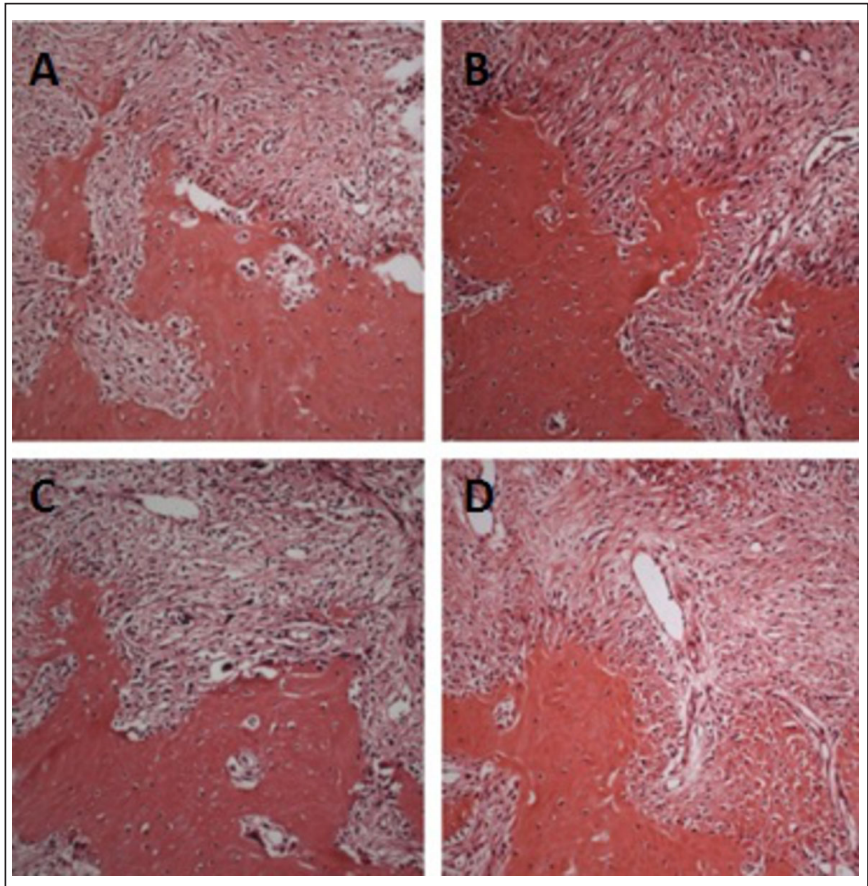

Fig. 1: Histological image of the left mandibular first molar furcation region with periodontal disease induced by ligature in rats after 7 days. (A) group OVZ with extraction; (B) group OVZ without extraction; (C) group SHAM with extraction; (D) group SHAM without extraction. All groups present similar aspects (HE; original magnification $\times 200$ ). were in contact with resorption lacunae in the periphery. The ligament attachment region showed slight inflammatory infiltration by mononuclear cells. By day 7 , a moderate mononuclear and polymorphonuclear inflammatory infiltrate was observed around the furcation area. In some specimens, migration of junctional epithelium was observed in this region, together with islands of remaining odontogenic epithelium. On days 3 and 7, bone trabeculae from the OVZ group were thinner than those of the SHAM group.

At 14 days, the bone septum of the furcation area of both groups was formed by thinner trabeculae, with smaller quantities of osteoclasts than in the previous periods. In several specimens from this period, it was observed the presence of junctional epithelium that migrated to the furcation area (Figure 2), with underlying moderate mononuclear inflammatory infiltrate. At 30 days, the bone septum of the furcation area was more compact, presenting fewer osteoclasts and less inflammatory infiltrate compared with earlier periods.

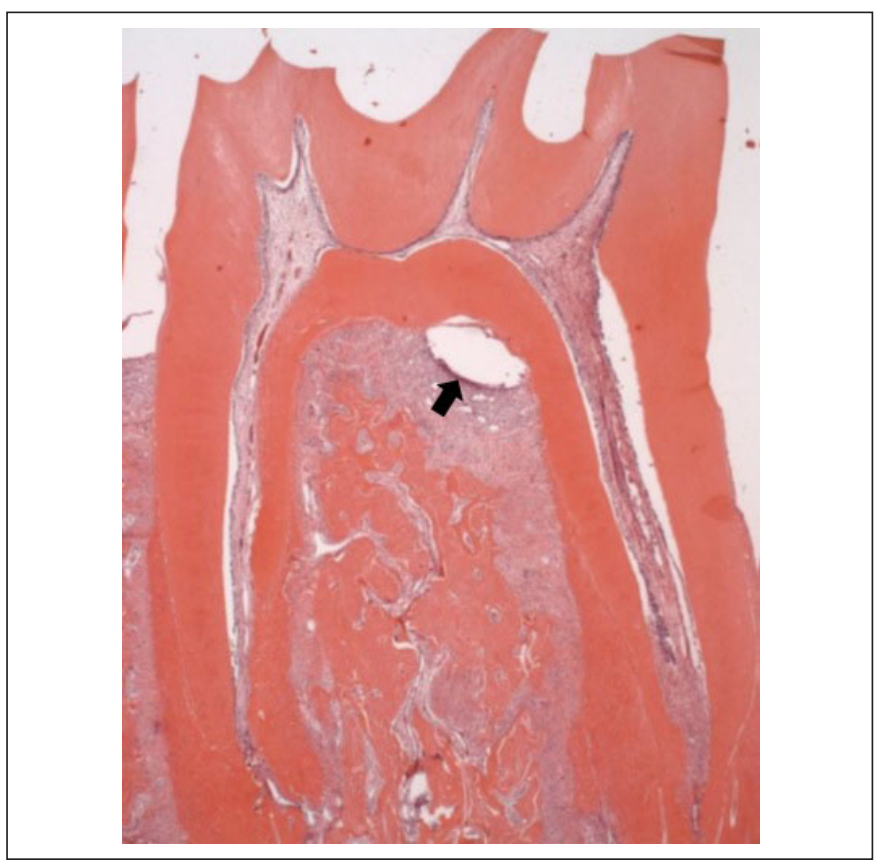

Fig. 2: (A) Histological image of the left mandibular first molar with periodontal disease induced by ligature. Note the migration of junctional epithelium (arrow) to the furcation area (group SHAM without extraction, after 14 days; HE; original magnification $\mathrm{x} 25)$.

\section{Histomorphometry of the furcation area}

The results presented (Figures 3,4) show the amount of bone present in the region of the furcation. The groups with a lower percentage of remaining bone matrix were those that displayed the greatest amount of bone resorption.

Analysis of the ANOVA test demonstrated statistically significant differences in the percentage of bone tissue when the variables presence or absence of estrogen and time of euthanasia were evaluated, $\mathrm{p}=0.020$ and $\mathrm{p}=0.002$, respectively. Post hoc Tukey significant difference analysis was used to determine significant differences among these variables (Figure 3). Concerning the extraction procedure, 


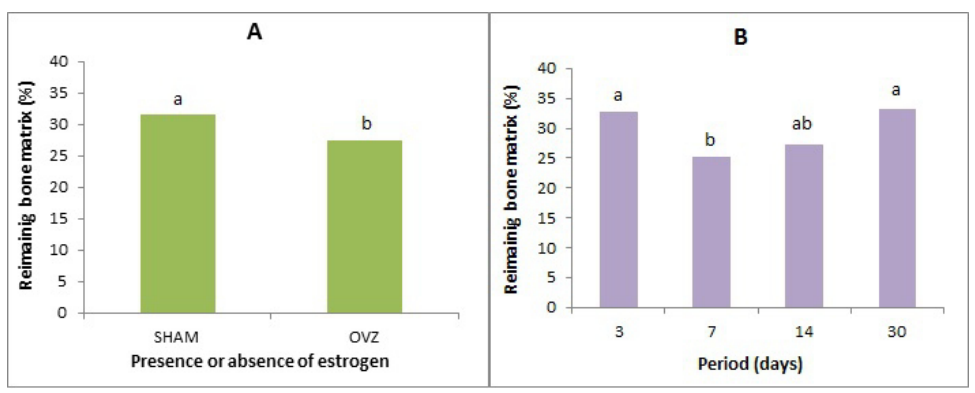

Fig. 3: Mean percentages of the remaining bone matrix (A): presence or absence of estrogen; (B): periods of euthanasia. Different letters indicate statistically significant difference $(p \leq 0.05)$.

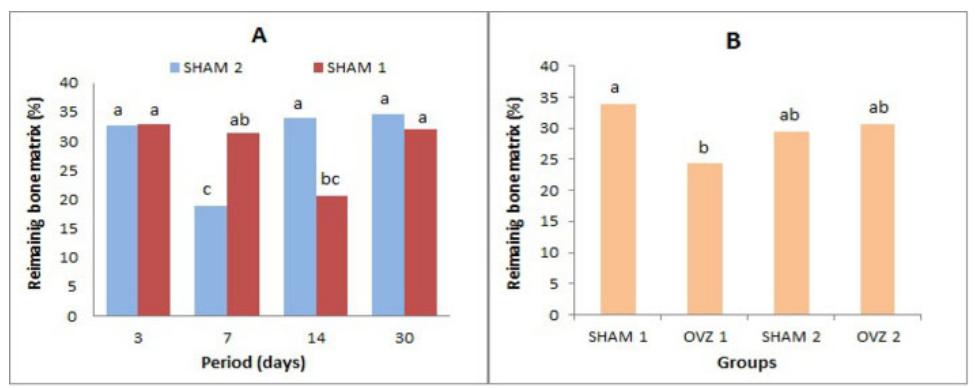

Fig. 4: Mean percentages of the remaining bone matrix (A): association between the variables time of euthanasia and extraction procedure; $(B)$ : association between the variables presence or absence of estrogen and extraction procedure. Different letters indicate statistically significant difference $(p \leq 0.05)$.

the ANOVA test demonstrated no statistically significant differences $(p=0.598)$.

When the association between the variables presence or absence of estrogen and time of euthanasia was analyzed, no statistically significant differences between the mean percentage of bone $(p=0.422)$ was observed. In contrast, when the association between time of euthanasia and the extraction procedure was analyzed, a statistically significant difference $(p=0.001)$ was observed. On day 30 and day 7 post periodontal disease induction, the groups that were submitted to antagonist tooth extraction exhibited the highest and lowest quantities of bone tissue, respectively (Figure $4 \mathrm{~A}$ ).

The association between the variables presence or absence of estrogen and extraction procedure showed a significant influence on the percentage of bone tissue $(p=0.003)$. However, the post hoc Tukey significant difference analysis indicated that the two groups that showed significant differences between them were SHAM and OVZ without extraction (SHAM 1 and OVZ 1) (Fig. 4 B).

The association among all three variables (presence or absence of estrogen, extraction procedure and time of euthanasia) showed no statistically significant difference $(p=0.083)$.

\section{Discussion}

In this study it was evaluated bone loss in furcation of rat teeth with induced periodontal disease under the influence of masticatory force and estrogen. Analysis of the results revealed that the absence of estrogen and the time course of periodontal disease are important factors for bone tissue resorption. However, masticatory force becomes relevant when combined with the time course of periodontal disease.

A well-established model for periodontal disease induction in rats was used, characterized by ligature placement around the cervical region of the mandibular first molar ${ }^{29}$. Plaque retention and trauma lead to a harmful inflammatory response that promotes greater susceptibility to periodontal disease $^{24-27}$

Based on the results obtained in this study, it was possible to verify that the presence of estrogen was a determining factor in the maintenance of bone tissue in teeth with periodontal disease. This is in agreement with previous studies indicating that osteoporosis, influenced by estrogen deficiency, is a recognized risk factor and may promote alveolar bone $\operatorname{loss}^{12-15}$.

A number of authors proposed that osteoporosis alone does not cause mandibular alveolar bone loss, stating that its combination with other osteopenic factors is required to induce significant bone $\operatorname{loss}^{18-19}$. Patullo et al. ${ }^{19}$ observed that the most important factor for bone loss is mandibular masticatory hypofunction, which causes harmful effects on bone quality in rats. Elovic et al..$^{20}$ observed that the extraction of molars from ovariectomized rats resulted in faster deterioration in the structural properties and greater reduction in area fraction of the jaw bone, compared with sham-operated rats. In the present study, when the combination between the variables presence or absence of estrogen and antagonist molar tooth extraction was analyzed, no significant increase in bone loss occurred in the furcation area. Greater bone loss was only observed in ovariectomized rats not submitted to antagonist molar tooth extraction compared with sham rats in the same conditions. It should be emphasized that the observed loss 
occurred specifically in alveolar bone with periodontal disease. Thus, the present results showed that the extraction procedure did not alter the quantity of bone in the furcation region in rats with periodontal disease, while estrogen deficiency is a major predisposing factor for bone loss.

In addition to the hormonal factor, alveolar bone resorption in the region of periodontal disease is also affected by time ${ }^{10,30}$. However, the period of greatest bone resorption following the induction of periodontal disease is controversial. In a study involving rats, Rodini et al. ${ }^{11}$ observed greater bone resorption from 3 to 7 days and resorption diminished from day 14 onward. Kuhr et al. ${ }^{30}$ observed higher bone resorption from days 1 to 15 and a decrease in bone loss from 30 to 60 days following periodontal disease induction.

In this study, 4 euthanasia times were investigated (3, 7, 14 and 30 days). The obtained results confirm the hypothesis that bone resorption is influenced by time, since the greatest resorption resulting from periodontal disease in ovariectomized rats, with or without masticatory force, generally occurred within the first 7 days. From day 14 onward, began a minor bone formation that became better defined by day 30 . These results are in agreement with those of previous studies ${ }^{11,30}$.

In conclusion, bone loss resulting from periodontal disease is increased by estrogen deficiency and varies according to the time course of periodontitis. In contrast, masticatory force does not seem to interfere in bone loss derived from periodontal disease. Further research is required to establish whether the reduction of masticatory force really does not interfere with bone loss resulting from periodontal disease in patients with and without osteoporosis.

\section{Acknowledgements}

The authors would like to thank to the Brazilian National Council for Scientific and Technological Development (PIBIC/CNPQ) for granting financial support.

\section{References}

1. Marques MR, Silva MAD, Barros SP. Periodontal disease and osteoporosis association and mechanism: A review of literature. Braz J Oral Sci. 2003; 2 : $137-40$.

2. Sandhu SK, Hampson G. The pathogenesis, diagnosis, investigation and management of osteoporosis. J Clin Pathol. 2011;64: 1042-50.

3. Govindarajan P, Böcker W, El Khassawna T, Kampschulte M, Schlewitz $G$, Huerter B et al. Bone matrix, cellularity, and structural changes in a rat model with high-turnover osteoporosis induced by combined ovariectomy and a multiple-Deficient Diet. Am J Pathol. 2014; 184: 765-77.

4. Häussler B, Gothe H, Göl D, Glaeske G, Pientka L, Felsenberg D. Epidemiology, treatment and costs of osteoporosis in Germany - the Bone EVAStudy. Osteoporos Int. 2007; 18: 77-84.

5. Lee YK, Yoon BH, Koo KH. Epidemiology of osteoporosis and osteoporotic fractures in South Korea. Endocrinol Metab (Seoul). 2013; 28: 90-3.

6. Dodd DZ, Rowe DJ. The relationship between postmenopausal osteoporosis and periodontal disease. J Dent Hyg. 2013; 87: 336-44.

7. Reddy MS. Oral osteoporosis: is there an association between periodontitis and osteoporosis? Compend Contin Educ Dent. 2002; 23: 21-8.
8. Kuo LC, Polson AM, Kang T. Associations between periodontal diseases and systemic diseases: A review of the inter-relationships and interactions with diabetes, respiratory diseases, cardiovascular diseases and osteoporosis. Public Health. 2008; 122: 417-33.

9. Nackaerts O, Horner K, Jacobs R, Karayianni K, Mitsea A, Berkas L et al. Is self reported alcohol consumption associated with osteoporotic mandibular bone loss in women? Eur J Oral Sci. 2009; 117: 7-12.

10. Viana LRF, Castro CP, Pereira HBW, Pereira AFV, Lopes FF. Is depression associated with periodontal status in elderly? Braz J Oral Sci. 2013; 12: $20-2$.

11. Rodini CO, Batista AC, Dionísio TJ, Santos CF, Cunha FQ, Lara VS. Morphologic evaluation and expression of matrix metalloproteinases-2 and 9 and nitric oxide during experimental periodontal disease in rat. J Mol Histol. 2008; $39: 275-82$.

12. Johnson RB, Gilbert JA, Cooper RC, Dai X, Newton BI, Tracy RR et al. Alveolar bone loss one year following ovariectomy in sheep. J Periodontol. 1997; 68: 864-71.

13. Lopes FF, Loureiro FH, Pereira Ade F, Pereira AL, Alves CM. Association between osteoporosis and periodontal disease. Rev Bras Ginecol Obstet. 2008; 30: 379-83.

14. Brennan-Calanan RM, Genco RJ, Wilding GE, Hovey KM, Trevisan M, Wactawski-Wende J. Osteoporosis and oral infection: independent risk factors for oral bone loss. J Dent Res. 2008; 87: 323-7.

15. Vishwanath SB, Kumar V, Kumar S, Shashikumar P, Shashikumar $Y$, Patel PV. Correlation of periodontal status and bone mineral density in postmenopausal women:A digital radiographic and quantitative ultrasound study. Indian J Dent Res. 2011; 22: 270-6.

16. Gomes-Filho IS, Passos J de S, Cruz SS, Vianna MI, Cerqueira E de M, Oliveira DC et al. The association between postmenopausal osteoporosis and periodontal disease. J Periodontol. 2007; 78: 1731-40.

17. Habashneh R, Alchalabi H, Khader YS, Hazza'a AM, Odat Z, Johnson GK. Association Between Periodontal Disease and Osteoporosis in Postmenopausal Women in Jordan. J Periodontol. 2010; 81: 1613-21.

18. Moriya $Y$, Ito K, Murai S. Effects of experimental osteoporosis on alveolar bone loss in rats. J Oral Sci. 1998; 40: 171-5.

19. Patullo IM, Takayama L, Patullo RF, Jorgetti V, Pereira RM. Influence of ovariectomy and masticatory hypofunction on mandibular bone remodeling. Oral Dis. 2009; 15: 580-6.

20. Elovic RP, Hipp JA, Hayes WC. Maxillary molar extraction causes increased bone loss in the mandible of ovariectomized rats. J Bone Miner Res. 1995; 10: 1087-93.

21. Ejiri S, Toyooka E, Tanaka M, Anwar RB, Kohno S. Histological and histomorphometrical changes in rat alveolar bone following antagonistic tooth extraction and/or ovariectomy. Arch Oral Biol. 2006; 51: 941-50.

22. Crespo Vázquez E, Crespo Abelleira A, Suárez Quintanilla JM, Rodriguez Cobos

23. MA. Correlation between occlusal contact and root resorption in teeth with periodontal disease. J Periodont Res. 2011; 46: 82-8.

24. Galvão MP, Chapper A, Rösing CK, Ferreira MB, de Souza MA. Methodological considerations on descriptive studies of induced periodontal diseases in rats. Pesq Odontol Bras. 2003; 17: 56-62.

25. Lohinai Z, Benedek P, Feher E, Györfi A, Rosivall L, Fazekas A et al. Protective effects of mercaptoethylguanidine, a selective inhibitor of inducible nitric oxide synthase, in ligature-induced periodontitis in the rat. $\mathrm{Br} \mathrm{J}$ Pharmacol. 1998; 123: 353-60.

26. Gaspersic R, Stiblar-Martincic D, Skaleric U. Influence of restraint stress on ligature-induced periodontitis in rats. Eur J Oral Sci. 2002; 110: 125-9.

27. Vasconcelos LMR, Ricardo LH, Balducci I, Vasconcellos LG, Carvalho YR. Histological analysis of effects of $24 \%$ EDTAgel for nonsurgical treatment of periodontal tissues. J Oral Sci. 2006; 48: 207-14.

28. Sallay K, Listgarten M, Sanavi F, Ring I, Nowotny A. Bacterial invasion of oral tissues of immunosuppressed rats. Infect Immun. 1984; 43: 1091-3.

29. Bentzen BH, Grauballe MCB, Björnsson MJ, Stoltze K, Hjørting Hansen E, Holmstrup P. A comparison of two models of experimental periodontitis in rats. Scand J Lab Anim Sci. 2005; 32: 73-80. 
30. Gaspersic R, Kovacic U, Cör A, Skaleric U. Unilateral ligature-induced periodontitis influences the expression of neuropeptides in the ipsilateral and contralateral trigeminal ganglion in rats. Arch Oral Biol. 2008; 53: 659-66.

31. Kuhr A, Popa-Wagner A, Schmoll H, Schwahn C, Kocher T. Observations on experimental marginal periodontitis in rats. J Periodontal Res. 2004; 39 : 101-6. 